205

\section{IDENTIFICATION OF SHUNTS BASED ON THE SHAPE OF DILUTION CURVE}

V. Kislukhin, N. Thuramalla, N. Krivitski

Transonic Systems Inc., Ithaca, NY, USA

Background and aims: The shape of dilution curve will dramatically change after indicator passes through the cardiopulmonary system in the presence of intra-cardiac shunts and a PDA. The purpose of this study was to develop an accurate algorithm for shunt identification and apply it to archived clinical data.

Methods: A mathematical model of indicator movement through cardiopulmonary system for three situations was developed:

1) No shunts;

2) Right to left shunt;

3) Left to right shunt.

Three parameters of dilution curve were examined:

1) appearance time;

2) asymmetry;

3) curve width.

Three possible scenarios were defined based on the probability " $P$ " of shunt identification:

1) No shunt, $P<5 \%$;

2) Possible shunt; $5 \%<\mathrm{P}<90 \%$;

3) Shunt identified $P>90 \%$ In the model, the values for cardiac output, heart size and heart rate were varied.

Results: In play-back mode the developed algorithm was applied to a clinical data archive of 1,188 dilution curves collected from adults $(n=131)$ and from children $(n=112)$ weighing between 0.9 - $130 \mathrm{~kg}$. Sensitivity of the method was $94 \%$, and false positive diagnostic was $0.9 \%$.

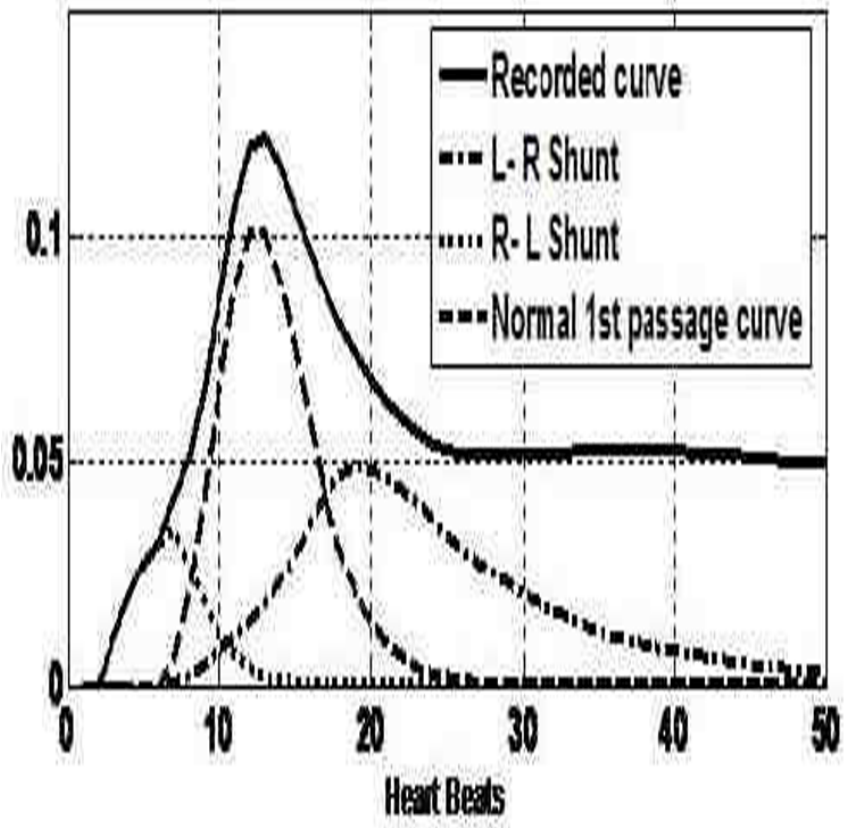

[Dilution Curve Shunt Model]

Conclusions: Automatic identification of shunts and PDA yielded acceptable accuracy. New PICU monitor (COstatus, Transonic Systems Inc., NY) in addition to measuring cardiac output and blood volumes, identifies the presence of intra-cardiac shunts based on developed algorithm. Next step will be to quantify the observed shunt flow value.

Grant support: NIH SBIR \# R44 HL061994

206

NT PRO-BNP, NGAL, AND H-FABP ARE INDICATORS OF HEMODYNAMIC RELEVANCE OF PATENT DUCTUS ARTERIOSUS IN PRETERM INFANTS

V. Tosse ${ }^{1}$, B. Hadzik ${ }^{1}$, H. Sabir ${ }^{1}$, P.E. Verde ${ }^{2}$, T. Hoehn ${ }^{1}$

${ }^{1}$ Neonatology and Pediatric Intensive Care, ${ }^{2}$ Coordination Center for Clinical Trials, University Hospital Duesseldorf, Duesseldorf, Germany

Background and aims: Patent ductus arteriosus (PDA) is the most common functional cardiovascular disease of preterm infants. Hemodynamic relevance for organs and circulation can neither be derived from clinical nor from echocardiographic criteria. The aim of the present study was to establish non-invasive parameters which reflect perfusiondependent consequences of the ductus for the organism of the preterm infant. 